\title{
Das Krebsleiden in der Medizin- und Kulturgeschichte
}

\author{
Dietrich von Engelhardt \\ Institut für Medizin- und Wissenschaftsgeschichte, Lübeck, Deutschland
}

\section{Kontext}

Krebs ist eine Krankheit von zentraler Bedeutung für Medizin, Kultur, Gesellschaft und vor allem den betroffenen Menschen, seine Familie und seine Freunde. Entstehung wie Erscheinung von Krebs sind vielen Menschen unheimlich und bedrohlich; trotz aller eindrucksvollen Erfolge in Diagnostik und Therapie steht die Medizin auch weiterhin vor Grenzen. Verzweiflung und Hoffnungslosigkeit sind aber keineswegs gerechtfertigt, notwendig ist die Differenzierung. Brustkrebs beispielsweise ist bei rechtzeitiger Erkennung (ohne Befall der Lymphknoten) durchaus erfolgreich zu behandeln, meistens kann auch die Brust erhalten bleiben.

Krebs ist eine alte Krankheit. Bösartige Neubildungen lassen sich seit Beginn der Menschheitsgeschichte nachweisen. Die Ausdrücke Krebs und Metastase stammen bereits aus der Antike. Krebs gehört zur Biologie und wird zugleich von der Kultur geprägt. Krebs stellt wie jede andere Krankheit eine körperliche, psychische, soziale und geistige Erscheinung dar und bedeutet immer ein Seins- und ein Werturteil, die Feststellung eines Faktums und seine normative Bewertung.

\section{Traditionen der Medizin}

Die medizinische Beschäftigung mit Krebs stand in der Antike und bis weit in die Neuzeit hinein unter dem Einfluss der Humoralpathologie, d.h. unter dem Konzept der Elemente, Qualitäten und Säfte. Demnach werden Krankheiten jeweils auf das pathogene Vorherrschen eines Saftes, eines Elementes oder einer Qualität zurückgeführt. Die Therapie muss entsprechend - neben den Interventionen der Chirurgie - die spezifische unangemessene und gefahrvolle Dominanz überwinden. Entscheidend im Spektrum der antiken Behandlung ist der Ansatz einer ganzheitlichen Diätetik, nicht im Sinne von Diät als einschränkender Planung des Essens und Trinkens, sondern umfassend als psychophysischer Umgang mit Licht und Luft, Bewegung und Ruhe, Essen und Trinken, Schlafen und Wachen, Ausscheidungen und Gefühlen. Zwischen den Extremen von Gesundheit und Krankheit gibt es den Bereich der Neutralität als dem normalen Zustand des Menschen, der weder je ganz gesund (= Unsterblichkeit) noch je ganz krank (= Tod) ist. Der Arzt für Freie beginnt nach Auffassung der Philosophen Plato und Aristoteles seine Therapie erst, wenn er sie dem Kranken erläutert und dieser ihr zugestimmt hat. Der Sklavenarzt ordnet dagegen wie ein Diktator ohne Erklärung und Empathie die Behandlung an. Der Arzt als medizinisch gebildeter Laie hält schließlich jeden Menschen für mitverantwortlich für seine Gesundheit, Krankheit und Therapie.

Im Mittelalter wird dieses kosmologisch-anthropologische Konzept in die religiöse Weltsicht eingefügt. Krankheit und Therapie erhalten einen transzendenten Sinn; hinter jedem Kranken steht die Passio Christi, hinter jedem Arzt Christus Medicus. Krankheit ist nicht nur eine biologische Zerstörung des Körpers, sondern hängt mit dem eschatologischen Weltverlauf vom Paradies über das irdische Leben zur Auferstehung zusammen, gehört unaufhebbar zur diesseitigen Existenz. Krankheit kann als Ausdruck der Erbsünde, der persönlichen Verfehlung, der Prüfung oder - was für Krebs allerdings weniger oder überhaupt nicht zutrifft - als Folge der Besessenheit verstanden werden. Aufklärung muss dem Sterbenden angeboten werden, damit dieser sich bewusst auf seinen eigenen Tod einstellen, von den Angehörigen Abschied nehmen und die irdischen Dinge zu Lebzeiten regeln kann. $\mathrm{Zu}$ Beginn der Neuzeit werden biochemische Theorien und entsprechende Behandlungsformen entwickelt. Hinzu kommen solidarpathologische Ansätze, die Gesundheit und Krankheit auf feste Bestandteile des Körpers, auf ihre normale oder abnormale Gestalt und Lage zurückführen. Chirurgi-

\begin{tabular}{ll}
\hline KARGER & ๑ 2007 S. Karger GmbH, Freiburg \\
Fax +49 7614520714 & Accessible online at: \\
$\begin{array}{l}\text { E-mail Information@Karger.de } \\
\text { www.karger.com }\end{array}$ & www.karger.com/onk
\end{tabular}

Prof. Dr. Dietrich von Engelhardt Institut für Medizin- und Wissenschaftsgeschichte Königstr. 42, 23552 Lübeck, Deutschland Tel. +49 451 707998-11 od. -12 (Sekr.), Fax -99 E-mail v.e@imwg.uni-luebeck.de 
sche Eingriffe werden fortgesetzt und zu verbessern gesucht, bleiben aber auf einem bescheidenen Stand. Säkularisierung, Naturalisierung und Individualisierung verändern das Kranken- wie Arztbild und auch ihre Beziehung, die heute nicht selten verkürzt wird zur Beziehung zwischen einem Dienstleistenden und einem Kunden oder zwischen einem Techniker und einer defekten Maschine.

Die zentrale Zäsur in der Geschichte der Onkologie fällt in das 19. Jahrhundert. Mit den Forschungen der Mediziner M.F.X. Bichat, Johannes Müller, Rudolf Virchow und anderen werden Gewebe und Zellen als entscheidende Ursachen des Krebses erfasst. Anästhesie und Antisepsis eröffnen ihrerseits fundamental neue Möglichkeiten für die Chirurgie. Das 20. Jahrhundert knüpft an diese Leistungen an und entwickelt eindrucksvolle neue diagnostische und therapeutische Verfahren.

Heute entfaltet sich das Spektrum der Therapie zwischen Chirurgie, Bestrahlung, Chemotherapie und auch Psychotherapie, umgreift Prävention, Kuration und Rehabilitation und schließt Supportivtherapie, Palliativstationen und Hospizinitiativen ein. Neben der objektiven wird auch die subjektive und soziale Seite der Krebserkrankung beachtet. Ein weiteres Charakteristikum der Gegenwart, auf das die empirisch-wissenschaftliche Medizin im Interesse des Kranken allerdings noch bessere Antworten als bislang geben muss, ist - gerade auch bei Krebserkrankungen - die verbreitete Hinwendung $\mathrm{zu}$ außerschulischen, auch irrationalen und abergläubischen Verfahren, die oft aus Enttäuschung über geringes Verständnis und eingeschränkte Kommunikation resultiert.

Durch die Geschichte hindurch haben sich Zeugnisse der Empfindungen, Ängste und Hoffnungen von Krebspatienten bis in die Gegenwart erhalten. Aus Scham und Angst verbirgt die gebildete Atossa (550-475 v. Chr.), Frau des persischen Herrschers Darius (549-486 v. Chr.), zunächst ihre Brustkrebserkrankung, wendet sich dann aber doch dem griechischen Sklavenarzt Demokedes zu, der sie nach der Überlieferung erfolgreich behandelt und zur Belohnung seine Freiheit erhält. Der römische Kaiser Diokletian (284-305) erkrankt an Kehlkopfkrebs, in der Renaissance Papst Innozenz VIII. (1432-1492) an Magenkrebs. Der Philosoph Pascal (16231662), für den «Krankheit der natürliche Zustand des Christen» ist, gewinnt im Glauben Kraft für die Bewältigung seines Magenkrebses. Die Konkurrenz der deutschen und englischen Ärzte in der Behandlung des Krebsleidens des Kaisers Friedrich III. (1831-1888) manifestiert die fatalen Auswirkungen, die Politik und persönlicher Ehrgeiz für die Medizin besitzen können.

Über Subjektivität und individuelle Situation des Krebskranken im 20. und 21. Jahrhundert informieren zahlreiche Selbsterfahrungstexte. Besondere Beachtung fanden Anne Philipes «Nur einen Seufzer lang» (franz. 1963), Fritz Zorns «Mars» (1979), Maxie Wanders «Leben wär' eine schöne Alternative» (1980), Brigitte Reimanns «Briefe und Tagebücher» (1983), Peter Nolls «Diktate über Sterben und Tod» (1984), Angelika
Mechtels «Jeden Tag will ich leben. Ein Krebstagebuch» (1990), Ruth Picardies «Es wird mir fehlen, das Leben» (engl. 1997), Ruth Pillats «Mein Krebs - mein Lehrmeister» (2005). Eindrucksvolle Berichte über Einstellung und Verhalten krebskranker Kinder enthalten die Monographien «Zur Psychologie der ausweglosen Situation» (1972) von Annemarie Wunnerlich, «Kinder sprechen vom Tod» (franz. 1980) von Ginette Raimbault, «Ich bin ja so allein - kranke Kinder zeichnen und sprechen über ihre Ängste» (1981) von Gisela Haas sowie aus neuerer Zeit «Wenn Kinder und Jugendliche sterben müssen» (2005) von Dietrich Niethammer.

Die Behandlung des Krebsleidens stellt die Onkologie immer wieder vor ethische und juristische Herausforderungen. Aufklärung ist zentral, ohne aufgeklärte Einwilligung (informed consent) bedeuten Diagnostik und Therapie für das geltende Recht in Deutschland Körperverletzung. Gegen den Willen muss aber andererseits niemand aufgeklärt werden; es gibt bei spezifischen Ausnahmen - auch ein Recht auf Nichtwissen, Kranke und Sterbende müssen sich auch anvertrauen können, ohne Vertrauen ist Medizin unmöglich. Aufklärung und Einwilligung entfalten sich in einer sozialen Situation und verlangen psychologische und kommunikative Fähigkeiten vom Arzt wie aber auch vom Kranken; sie entsprechen etabliertem Brauch, sind Gebote der medizinischen Standesethik, sind ein Beweis ärztlicher Kultur in der Onkologie.

Aufklärung muss nach den Dimensionen: Diagnose, Ursachen, Prognose, Therapie und sozialpsychologische Folgen der Krankheit für das Leben unterschieden werden. Aufklärung sollte ein abgestuftes Angebot zwischen eingeschränkter Information und Solidarität in der Wahrheit der Situation sein wie viele Stufen dieser «Treppe der Aufklärung» hinaufgeschritten wird, hat im Prinzip der Krebspatient, solange er vernünftig und bei Bewusstsein ist, selbst zu bestimmen.

Ebenso entscheidend für die Onkologie ist der Umgang mit Sterben und Tod. Aller medizinischer Fortschritt wird die Grundbedingung des menschlichen Lebens nicht aufheben können: ein Ende zu haben und um dieses Ende auch zu wissen. Auch die Medizin muss diese Grundparadoxie anerkennen: heilen zu wollen, was letztlich nicht zu heilen ist. Nur zu schmerzlich erfährt der Arzt im Umgang mit dem sterbenden Patienten die Grenzen seines Wissens und seines Könnens; innere Euthanasie als guter und schmerzfreier Tod oder als Sterbebegleitung gewinnt in dieser Perspektive seine wahre Bedeutung und notwendige Funktion.

Neben aktiver und passiver Euthanasie sind direkte und indirekte Euthanasie, arztassistierter Suizid, äußere und innere Euthanasie sowie Anlass der Euthanasie zu unterscheiden. In den Niederlanden und Belgien ist unter gewissen Bedingungen (Freiwilligkeit, Aufklärung, unerträgliches und irreversibles Leiden, Hinzuziehung eines zweiten Arztes, sorgfältige Durchführung, Meldung an Leichenbeschauer, Staatsanwalt und Ethikkommission) aktive Euthanasie als Tötung möglich. Den Überlegungen, wie Sterbebegleitung als innere oder geistige Euthanasie im ursprünglichen Wortsinn (griech. $e u=$ 
schön und gut; thanatos $=$ Tod) in der Medizin wie allgemein im Leben verwirklicht werden kann, kommt quantitativ und qualitativ ohne Zweifel eine größere Bedeutung zu als allen Diskussionen über die Legalisierung der aktiven Euthanasie als Beendigung des Lebens. Gelingt bei guter Schmerztherapie eine seelisch-geistige Begleitung des Sterbenden, wird die Bitte um Lebensverkürzung oft gar nicht erst entstehen.

\section{Die Perspektive der Künste und Literatur}

Krebs ist nicht nur ein Thema der Medizin, Krebs hat wiederholt zu Darstellungen und Deutungen in der Theologie und Philosophie sowie vor allem in den Künsten und der Literatur geführt. Onkologie verbindet Naturwissenschaften und Geisteswissenschaften, ist Wissenschaft und Kunst. Das Interesse galt dabei nicht nur der objektiven Phänomenologie und physischen Ätiologie der Krankheit, sondern stets auch der Subjektivität des Kranken, nicht allein dem Arzt, seiner Therapie und dem Krankenhaus, sondern ebenso den sozialen Reaktionen und vor allem der geistig-symbolischen Bedeutung des Krebsleidens. Die Anzahl literarischer Texte ist groß; besonders intensiv wurde Krebs in Erzählungen und Romanen des 19. und 20. Jahrhunderts aufgegriffen.

Objektivität und Subjektivität der Krebserkrankung sowie die enttäuschenden Reaktionen der Ärzte und Angehörigen werden in L.N. Tolstojs Erzählung «Der Tod des Iwan Iljitsch» (russ. 1886) detailliert wiedergegeben: «Diese Lüge um ihn und in ihm vergiftete mehr als alles die letzten Lebenstage von Iwan Iljitsch.» Die Ärzte sind desinteressiert, mitleidslos auch in ihrem aufmunternden Zuspruch, die Diagnose der Krankheit ist ihnen wichtiger als die Leidenssituation des Kranken; für sie gibt es «nur einen Streit zwischen Wanderniere und Blinddarm». Kurz vor dem Tode erfasst Ivan Iljitsch aber in dem Nichts, das er auf sich zukommen fühlt, eine Wahrheit, eine beruhigende und tragende Substanz: « Ja, es war alles nicht das Wahre>, sagte er zu sich, doch das macht nichts. Man kann ja, noch kann man es erreichen, das 〈Wahre〉. Doch was ist das 〈Wahre〉?» Der Schmerz schwindet, es vergeht die Todesangst, ein Licht erscheint ihm: « So ist das also!〉, sagte er plötzlich laut. 〈Welche eine Freude!〉»

Immer wieder wird auch in der Literatur die Krebserkrankung nicht oder zu spät erkannt oder falsch gedeutet. Thomas Manns späte Erzählung «Die Betrogene» (1953) schildert eine Frau im Alter von etwa 50 Jahren, die ihre Blutungen aufgrund eines Unterleibkrebses für eine Rückkehr ihrer Menstruation hält und sich in dieser Situation in einen 24-jährigen Amerikaner verliebt. Die Täuschung durch die Natur wird von ihr aber, wie sie zu ihrer Tochter kurz vor ihrem Ende sagt, akzeptiert und sogar bejaht: «Aber wie wäre denn Frühling ohne den Tod? Ist ja doch der Tod ein großes Mittel des Lebens, und wenn er für mich die Gestalt lieh von Auferstehung und Liebeslust, so war das nicht Lug, sondern Güte und Gnade.»
Die Ansichten der literarischen Ärzte über die Ursachen des Krebses können von den Auffassungen ihrer zeitgenössischen Kollegen in der Realität nicht unerheblich abweichen. Graham Greenes «Doktor Crombie» (engl. 1956) ist von der sexuellen Ätiologie des Krebses zutiefst überzeugt und versucht die Schüler des unter dem zügellosen englischen König Heinrich VIII. (!) gegründeten Gymnasiums in diesem Sinne in seinen Aufklärungsvorträgen zur Keuschheit zu bewegen. Einer seiner ehemaligen Schüler, der sich viermal verheiratet hat, erkrankt mit über 60 Jahren an Lungenkrebs und erinnert sich bei dieser Gelegenheit an den Schularzt: «Natürlich führen die Ärzte mein Leiden auf mein übermäßiges Zigarettenrauchen zurück; trotzdem belustigt es mich, mit Dr. Crombie zu glauben, daß es durch übermäßigen Genuß einer wesentlich angenehmeren Art hervorgerufen wurde.»

Wahrheit und Lüge können auch das Verhältnis zwischen dem Krebskranken und seinen Angehörigen oder Freunden bestimmen. Robert und Kate in J.A. Philipps' Erzählung «Andenken» (engl. 1979), verheimlichen der Mutter aus Mitleid ihre Diagnose Gehirntumor. Während des Krankenhausaufenthaltes nimmt die Tochter die Mutter zu einem Besuch auf dem Rummelplatz mit. Auf der höchsten Stelle des Riesenrades erweist sich ihre schonende Lüge als unnötig: «IIch weiß genau Bescheid , sagte ihre Mutter, <ich weiß, was du mir verschwiegen hast.> Über ihnen spannte sich der graue Himmel, war unaufhaltsam in Bewegung. Kate saß ganz ruhig da und schluckte. Unablässig fixierten sie einander. Sie sah sich selbst in den braunen Augen ihrer Mutter und hatte das Gefühl, als würde sie ganz langsam in sie hineinfallen.»

Was Operationen in der Vergangenheit bedeutet haben, kann man sich heute kaum noch vorstellen. In Samuel Warrens Erzählung «Krebs» (engl. 1830) wird von einer Brustamputation berichtet, die in jener Zeit des 19. Jahrhunderts noch ohne Anästhesie durchgeführt werden musste. Die Patientin gewinnt Trost und Kraft durch die Lektüre der Liebesbriefe ihres Mannes, dem sie aus Rücksicht auf sein Empfinden den Eingriff verschwiegen hat: «Ihre Augen blieben unausgesetzt mit einem Hinblick glühender Zärtlichkeit auf die teuren Schriftzüge ihres Gatten geheftet; sie bewegte während der ganzen schmerzhaften, sich lange hinzögernden, Operation kein Glied, und nur ein leises Seufzen wurde zuweilen von ihr vernommen.» Mit der Entwicklung der physischen Anästhesie hat sich die hilfreiche Bedeutung der «spirituellen Anästhesie» durch Literatur und Künste fast verloren, wird in der Gegenwart aber zunehmend wieder anerkannt und aufgegriffen.

In Carson McCullers' Roman «Uhr ohne Zeiger» (engl. 1961) gelangt der krebskranke Apotheker Malone (wohl mit dem Sinn: I am alone) zur Annahme seines Todes und erfährt die liebevolle Zuwendung seiner Frau Martha. «Er war ein Mann, der eine Uhr ohne Zeiger beobachtet.» Von der Aufklärung hatte er sich Beruhigung und nicht Wahrheit erhofft: «Er hatte Mitgefühl und Beruhigung verlangt, und statt dessen bekam er sein Todesurteil zu hören.» Im Krankenhaus stößt 
«Je désirais to voir. J'ai peur de mourir ici.» «Est-ce bien toi?»
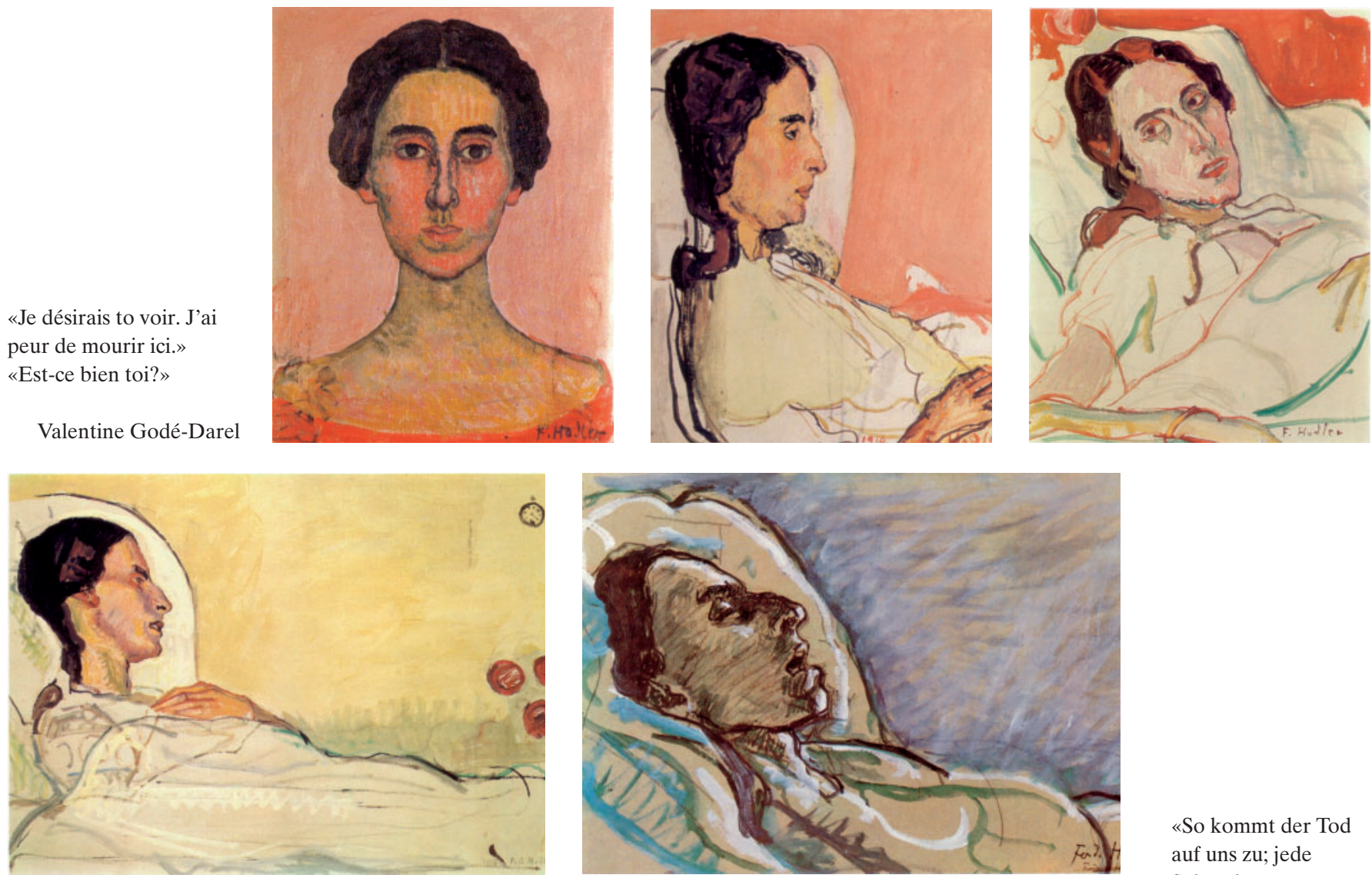

«So kommt der Tod auf uns zu; jede Sekunde unseres
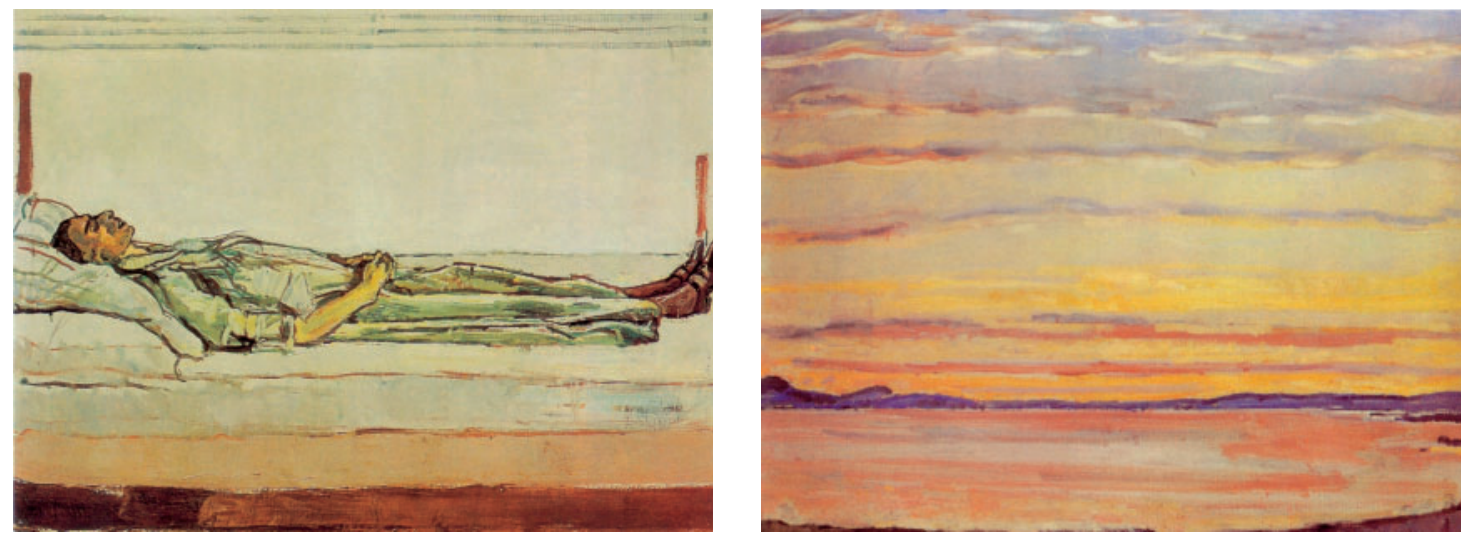
Lebens ist es eine schöne, ruhige Bewegung und eine Gegenbewegung. Das gliedert unser ganzes Leben; es gibt ihm einen vollkommen anderen Rhythmus! Das zu wissen, das verwandelt den Todesgedanken in eine gewaltige Kraft.»

Ferdinand Hodler

Abb. 1. Oben: «Rotes» Bildnis Valentine Godé-Darel um 1910; die kranke Valentine Godé-Darel, beide 1914. Mitte: Die kranke Valentine GodéDarel, 1914; die sterbende Valentine Godé-Darel, 1915. Unten: Die tote Valentine Godé-Darel, 26. Januar 1915, Sonnenuntergang am Genfersee, 1915. Ferdinand Hodler.

Malone auf die Welt der Bücher, vor allem fasziniert ihn ein Buch mit dem Titel «Krankheit zum Tode» von Sören Kierkegaard, in dem er auf einen Satz stößt: «Die größte Gefahr sein Ich zu verlieren - kann sich so still vollziehen, als wäre es nichts; jeder andere Verlust - von einem Arm oder Bein, von fünf Dollar, von einer Ehefrau und so weiter - fällt einem bestimmt auf.» Die tödliche Krankheit konfrontiert Malone mit der Existenz des Menschen und vor allem mit seiner eigenen
Existenz und ihrer Endlichkeit und wird zugleich zu einer Erweiterung seines Bewusstseins, ist bei allem Schmerz und aller Trauer auch ein Gewinn: «... das Sterben hatte sein Daseinsgefühl gesteigert, während er im Krankenbett lag und das leuchtende Blut Tropfen für Tropfen niedertröpfeln sah.»

Empathie und Zuwendung können den Tod überdauern. Bis zur Selbstaufgabe nimmt der 11-jährige Paul in Jean-Edern Halliers Roman «Der zuerst schläft, weckt den anderen» 
(franz. 1977) am Schicksal seines an einem Gehirntumor erkrankten 12-jährigen Bruders Aubert Anteil. «So leidenschaftlich war ich Aubert, daß ich oft beinahe in ihn hinübersank und unwiderstehlich vom Sog seines eigenen Leidens gepackt, mich in seinen Abgrund verlor.» Der Sterbende schenkt dem Lebenden Wahrheit, dieser führt das Leben des Verstorbenen fort, der Tod gehört zum Leben.

Kranker und Arzt, Therapie und medizinische Institution weisen über sich hinaus, werden zu Metaphern und Symbolen. In Solschenizyns Roman «Die Krebsstation» (russ. 1968), die nicht nur für Russland, sondern für die ganze Welt steht, findet sich eine Vielfalt von Krebserkrankungen mit unterschiedlichen individuellen Verhaltensweisen, sozialen Reaktionen und therapeutischen Interventionen. Die Ärzte sind Therapeuten und Forscher, sie können, wie sich am Schicksal von Ljudmila Afanasejevna Donzova erweist, selbst an Krebs erkranken. Die Therapie besteht in Hormontherapie, Bestrahlung, Operation, Behandlung mit Kolloidgold. Grenzen werden gesehen und anerkannt, stets muss ein Ausgleich zwischen Beistand, Therapie und Forschung gefunden werden.

Die Problematik der aktiven Euthanasie thematisiert Theodor Storm in der Erzählung «Ein Bekenntnis» (1888). Der Arzt erlöst und tötet in dieser Novelle seine eigene krebskranke Frau auf ihre Bitte hin und muss später erfahren, dass er eine neue Therapiemöglichkeit übersehen hat, die sie vielleicht hätte retten können. Im professionellen Versagen geht ihm «die Heiligkeit des Lebens» auf, die ihm zufolge der Medizin und jeder aktiven Beendigung des Lebens unüberschreitbare Grenzen setzen sollte: «... nach dem Mysterium soll kein Mensch, kein Mann der Wissenschaft seine Hand ausstrecken, wenn er's nur tut im Dienst des Todes, denn sie wird ruchlos gleich der des Mörders.»

Spektakuläre und auch utopische oder in die Zukunft weisende Fortschritte in der Onkologie werden vor allem in der Science-Fiction-Literatur beschrieben. Das Einfrieren eines Krebskranken in Leonard Tushnets «In re Glover» (engl. 1972) zieht juristische, medizinische und soziale Probleme in der Bestimmung des Todes nach sich, die ihre Lösung durch das versehentliche Auftauen des nun endgültig toten Körpers finden. Um der Agonie des Krebsleidens zu entgehen, setzt in Kurt Vonneguts «Katzenwiege» (engl. 1963) der Diktator «Papa» Monzano, betreut von dem ehemaligen Lagerarzt in Auschwitz Dr. Schlichter von Koenigswald, mit einem geheimnisvollen Eiskristall seinem Leben ein Ende; durch unglückselige Umstände löst dieser Selbstmord dann aber die Vereisung der ganzen Welt aus.

Onkologie und Kultur heißt auch Verfilmungen der Krebserkrankung, nicht selten auf der Grundlage literarischer Vorlagen. Ebenso finden sich mehrfach Wiedergaben in der abbildenden Kunst. Ob Raffael auf seinem Gemälde «Fornarina» (1518) allerdings wirklich einen Brustkrebs darstellen wollte, bleibt umstritten. Ein bewegendes Beispiel verbindet sich mit dem Schweizer Maler Ferdinand Hodler, der in einem großen Zyklus von Zeichnungen und Bildern das Kranksein und Ster- ben seiner Freundin Valentine Godé-Darel in den Jahren 1913-1915 festgehalten hat (Abb. 1). Zugleich wird in diesen Bildern die Kraft offenkundig, die Kunst dem Künstler, dem Leidenden und allen Menschen verleihen kann; am 14.11.1914 notiert sich der Maler in sein Tagebuch: «So kommt der Tod auf uns zu; jede Sekunde unseres Lebens ist es eine schöne, ruhige Bewegung und eine Gegenbewegung. Das gliedert unser ganzes Leben; es gibt ihm einen vollkommen anderen Rhythmus! Das zu wissen, das verwandelt den Todesgedanken in eine gewaltige Kraft.»

$\mathrm{Zu}$ Krebs als Phänomen der Kulturgeschichte gehört auch seine Verwendung in Redewendungen, Sprichwörtern, ebenso auch als Metapher und Symbol. Von des «Aberglaubens Krebs, der viele Lehrer plagt», spricht Friedrich von Hagedorn. In Leidenschaften sieht der Philosoph Kant «Krebsschäden für die reine praktische Vernunft und mehrenteils unheilbar» (1798). In Jean Pauls Hesperus (1795) ist vom «philosophischen Krebsgifte» die Rede, hier wird ebenfalls zu bedenken gegeben: «Der eifersüchtige Krebs auf der Brust (= Krebs der Eifersucht) ist nie ganz zu schneiden, wenn ich großen Heilkünstlern glauben soll.» Charles Baudelaire stellt 1852 fest: «Eine frenetische Kunstleidenschaft ist ein Krebs, der den Rest verschlingt.» Der Futurist Marinetti meint 1920: «Der Kommunismus ist die Verschärfung der bürokratischen Krebskrankheit, die stets jede Menschlichkeit aufgezehrt hat.»

\section{Ausblick}

Krebs ist ein zentrales Thema der Medizin- und Kulturgeschichte, der Natur wie der Kultur. Medizin ist selbst Kunst, wie auch die Künste therapeutische Kraft besitzen. «Die Kunst ist lang, das Leben kurz», heißt es bei allen Fortschritten in Diagnostik und Therapie auch heute noch zutreffend in einem hippokratischen Aphorismus aus der Antike.

Krebs gehört - wie Geisteskrankheit - zu den Krankheiten, an denen das weite und komplexe Spektrum zwischen Objektivität und Subjektivität, zwischen Phänomenologie und Symbolik auf besonders tiefgründige Weise manifest wird. Krebs ist Ausdruck der destruktiven Autonomie des Körpers, die dem Betroffenen subjektiv über längere Zeit verborgen bleiben und sogar in den körperlichen Auswirkungen Gesundheit vortäuschen kann.

In der Literatur und den Künsten wird die Krebserkrankung in einer Vielfalt von Dimensionen dargestellt und gedeutet in den physischen, psychischen, sozialen und geistigen Dimensionen, in dem Dreiecksverhältnis zwischen Patient, Arzt und Gesellschaft, in den Möglichkeiten und Grenzen der Medizin, in der Verbindung von überzeitlichen und zeitbezogenen Zügen. Die Welt der Krankheit und Medizin kann auf Metaphern und Symbole, Redewendungen und Sprichwörter nicht verzichten, die allerdings auch belasten und irreführende Interpretationen nahe legen können, wofür der Krebs seinerseits ein zutreffendes Beispiel ist. 
In der Perspektive der Medizin- und Kulturgeschichte wird Krebs immer wieder zu einem eindrucksvollen Beweis des ärztlichen Engagements, der Lebens- und Sterbekunst, der mitmenschlichen Solidarität. Literatur und Künste entwerfen neben Philosophie und Theologie Vorbilder, Leitideen und Perspektiven, die dem Betroffenen und seinen Angehörigen wie den Ärzten und Pflegepersonen Anregung und Orientierung, Hilfe und Trost geben können, die an die Ganzheitlichkeit des Krankseins, an die Verbindung von Natur und Kultur erinnern. In dieser Perspektive kann zuversichtlich und mit Hoffnung auf die weitere Entwicklung der Onkologie gesetzt werden.

\section{Weiterführende Literatur}

Eckart WU (Hrsg): 100 Years of Organized Cancer Research - 100 Jahre organisierte Krebsforschung. Stuttgart, Thieme, 2000.

von Engelhardt D: Medizin in der Literatur der Neuzeit, vol 1-2. Hürtgenwald, Pressler, 1991/2000.

Förster F (Hrsg): Recht und Ethik in Onkologie und Palliativmedizin: Rahmenbedingungen für die Partnerschaft zwischen Arzt und Patient. Fachkrankenhaus Marienstift Schwarzenberg, 2003.

von Jagow B, Steger F (Hrsg): Literatur und Medizin. Ein Lexikon. Göttingen, Vandenhoeck und Ruprecht, 2005.

Moamai M: Krebs schreiben. Deutschsprachige Literatur der siebziger und achtziger Jahre. St. Ingbert, Rohrig, 1997.

Moore RJ, Spiegel D: Cancer, Culture, and Communication. New York, Kluwer/Plenum, 2004.

Spross JA: Cancer pain and suffering: Clinical lessons from life, literature and legend. Oncol Nurs Forum 1985;12:23-31. 